\title{
Existence of a solution of integral equations via fixed point theorem
}

\author{
Selma Gülyaz ${ }^{1}$, Erdal Karapınar²* Vladimir Rakocević 3 and Peyman Salimi ${ }^{4}$
}

\section{"Correspondence:}

erdalkarapinar@yahoo.com;

ekarapinar@atilim.edu.tr

${ }^{2}$ Department of Mathematics,

Atilim University, İncek, Ankara 06836, Turkey

Full list of author information is available at the end of the article

\section{Abstract}

In this paper, we establish a solution to the following integral equation:

$$
u(t)=\int_{0}^{T} G(t, s) f(s, u(s)) d s \quad \text { for all } t \in[0, T],
$$

where $T>0, f:[0, T] \times \mathbb{R} \rightarrow \mathbb{R}$ and $G:[0, T] \times[0, T] \rightarrow[0, \infty)$ are continuous functions. For this purpose, we also obtain some auxiliary fixed point results which generalize, improve and unify some fixed point theorems in the literature.

MSC: $47 \mathrm{H} 10 ; 54 \mathrm{H} 25$

Keywords: cyclic representation; fixed point

\section{Introduction and preliminaries}

Fixed point theory is one of the most efficient tools in nonlinear functional analysis to solve the nonlinear differential and integral equations. The existence/uniqueness of a solution of differential/integral equations turns into the existence/uniqueness of a (common) fixed point of the operators which are obtained after suitable substitutions and elementary calculations; see, e.g., [1-14].

In this paper, we first obtain some fixed point theorems to solve the integral equation mentioned above. For the sake of completeness, we recollect some basic definitions and elementary results. Let $X$ be a nonempty set and $T$ be a self-mapping on $X$. Then, the set of all fixed points of $T$ on $X$ is denoted by $\operatorname{Fix}(T)_{X}$. Let $\Psi$ be the set of all functions $\psi:[0, \infty) \times[0, \infty) \rightarrow[0, \infty)$ satisfying the following conditions:

(1) $\psi$ is continuous,

(2) $\psi\left(t_{1}, t_{2}\right)=0$ if and only if $t_{1}=t_{2}=0$,

(3) $\psi\left(t_{1}, t_{2}\right) \leq \frac{1}{2}\left(t_{1}+t_{2}\right)$.

Cyclic mapping and cyclic contraction were introduced by Kirk-Srinavasan-Veeramani to improve the well-known Banach fixed point theorem. Later, various types of cyclic contraction have been investigated by a number of authors; see, e.g., [6, 15-17].

Definition 1.1 [18] Suppose that $(X, d)$ is a metric space and $T$ is a self-mapping on $X$. Let $m$ be a natural number and $X_{i}, i=1, \ldots, m$, be nonempty sets. Then $Y=\bigcup_{i=1}^{m} X_{i}$ is called a

\section{囪 Springer}

( 2013 Gülyaz et al.; licensee Springer. This is an Open Access article distributed under the terms of the Creative Commons Attribution License (http://creativecommons.org/licenses/by/2.0), which permits unrestricted use, distribution, and reproduction in any medium, provided the original work is properly cited. 
cyclic representation of $X$ with respect to $T$ if

$$
T\left(X_{1}\right) \subset X_{2}, \quad \ldots, \quad T\left(X_{m-1}\right) \subset X_{m}, \quad T\left(X_{m}\right) \subset X_{m+1},
$$

where $X_{m+1}=X_{1}$.

Definition 1.2 [17] Let $T: X \rightarrow X, r>0$ and $\eta, \xi: X \rightarrow[0,+\infty)$ be two functions. We say that $T$ is $r-(\eta, \xi)$-admissible if

(i) $\eta(x) \geq r$ for some $x \in X$ implies $\eta(T x) \geq r$,

(ii) $\xi(x) \leq r$ for some $x \in X$ implies $\xi(T x) \leq r$.

Definition 1.3 Let $(X, d)$ be a metric space and $T: Y \rightarrow Y$ be a self-mapping, where $Y=$ $\bigcup_{i=1}^{m} X_{i}$ is a cyclic representation of $Y$ with respect to $T$. Let $\eta, \xi: Y \rightarrow[0,+\infty)$ be two functions. An operator $T: Y \rightarrow Y$ is called:

- a cyclic weak $r-(\eta, \xi)$-C-contractive mapping of the first kind if

$$
\eta(x) \eta(y) d(T x, T y) \leq \xi(x) \xi(y)\left[\frac{1}{2}[d(x, T y)+d(y, T x)]-\psi(d(x, T y), d(y, T x))\right]
$$

holds for all $x \in X_{i}$ and $y \in X_{i+1}$, where $\psi \in \Psi$.

- a cyclic weak $r-(\eta, \xi)$-C-contractive mapping of the second kind if

$$
[\eta(x) \eta(y)+r]^{d(T x, T y)} \leq[\xi(x) \xi(y)+r]^{\left[\frac{1}{2}[d(x, T y)+d(y, T x)]-\psi(d(x, T y), d(y, T x))\right]}
$$

such that $r^{2}+r>1$ holds for all $x \in X_{i}$ and $y \in X_{i+1}$, where $\psi \in \Psi$.

\section{Auxiliary fixed point results}

We state the main result of this section as follows.

Theorem 2.1 Let $(X, d)$ be a complete metric space, $m \in \mathbb{N}, X_{1}, X_{2}, \ldots, X_{m}$ be nonempty closed subsets of $(X, d)$ and $Y=\bigcup_{i=1}^{m} X_{i}$. Suppose that $T: Y \rightarrow Y$ is a cyclic weak $r-(\eta, \xi)$ $C$-contractive mapping of the first kind such that

(i) $T$ is $r-(\eta, \xi)$-admissible;

(ii) there exists $x_{0} \in Y$ such that $\eta\left(x_{0}\right) \geq r$ and $\xi\left(x_{0}\right) \leq r$;

(iii) if $\left\{x_{n}\right\}$ is a sequence in $Y$ such that $\eta\left(x_{n}\right) \geq r$ and $\xi\left(x_{n}\right) \leq r$ for all $n \in \mathbb{N}$ and $x_{n} \rightarrow x$ as $n \rightarrow \infty$, then $\eta(x) \geq r$ and $\xi(x) \leq r$.

Then $T$ has a fixed point $x \in \bigcap_{i=1}^{n} X_{i}$. Moreover, if $\eta(x) \geq r, \eta(y) \geq r, \xi(x) \leq r, \xi(y) \leq r$ for all $x, y \in F i x(T)_{Y}$, then $T$ has a unique fixed point.

Proof Let there exist $x_{0} \in Y$ such that $\eta\left(x_{0}\right) \geq r$ and $\xi\left(x_{0}\right) \leq r$. Since $T$ is $r-(\eta, \xi)$ admissible, then $\eta\left(T x_{0}\right) \geq r$ and $\xi\left(T x_{0}\right) \leq r$. Again, since $T$ is $r-(\eta, \xi)$-admissible, then $\eta\left(T^{2} x_{0}\right) \geq r$ and $\xi\left(T^{2} x_{0}\right) \leq r$. By continuing this process, we get

$$
\eta\left(T^{n} x_{0}\right) \geq r \quad \text { and } \quad \xi\left(T^{n} x_{0}\right) \leq r \quad \text { for all } n \in \mathbb{N} .
$$

On the other hand, since $x_{0} \in Y$, there exists some $i_{0}$ such that $x_{0} \in X_{i_{0}}$. Now $T\left(X_{i_{0}}\right) \subseteq$ $X_{i_{0}+1}$ implies that $T x_{0} \in X_{i_{0}+1}$. Thus there exists $x_{1}$ in $X_{i_{0}+1}$ such that $T x_{0}=x_{1}$. Similarly, 
$T x_{n}=x_{n+1}$, where $x_{n} \in X_{i_{n}}$. Hence, for $n \geq 0$, there exists $i_{n} \in\{1,2, \ldots, m\}$ such that $x_{n} \in X_{i_{n}}$ and $x_{n+1} \in X_{i_{n}+1}$. In case $x_{n_{0}}=x_{n_{0}+1}$ for some $n_{0}=0,1,2, \ldots$, then it is clear that $x_{n_{0}}$ is a fixed point of $T$. Now assume that $x_{n} \neq x_{n+1}$ for all $n$. Hence, we have $d\left(x_{n-1}, x_{n}\right)>0$ for all $n$. Set $d_{n}:=d\left(x_{n}, x_{n+1}\right)$. We shall show that the sequence $\left\{d_{n}\right\}$ is non-increasing. Due to (2) with $x=x_{n-1}$ and $y=x_{n}$, we get

$$
\begin{array}{rl}
r^{2} & d\left(x_{n}, x_{n+1}\right) \\
& =r^{2} d\left(T x_{n-1}, T x_{n}\right) \leq \eta\left(x_{n-1}\right) \eta\left(x_{n}\right) d\left(T x_{n-1}, T x_{n}\right) \\
& \leq \xi\left(x_{n-1}\right) \xi\left(x_{n}\right)\left[\frac{1}{2}\left[d\left(x_{n-1}, T x_{n}\right)+d\left(x_{n}, T x_{n-1}\right)\right]-\psi\left(d\left(x_{n-1}, T x_{n}\right), d\left(x_{n}, T x_{n-1}\right)\right)\right] \\
& =\xi\left(x_{n-1}\right) \xi\left(x_{n}\right)\left[\frac{1}{2} d\left(x_{n-1}, x_{n+1}\right)-\psi\left(d\left(x_{n-1}, x_{n+1}\right), 0\right)\right] \\
& \leq r^{2}\left[\frac{1}{2} d\left(x_{n-1}, x_{n+1}\right)-\psi\left(d\left(x_{n-1}, x_{n+1}\right), 0\right)\right],
\end{array}
$$

which implies

$$
\begin{aligned}
d\left(x_{n}, x_{n+1}\right) & \leq \frac{1}{2} d\left(x_{n-1}, x_{n+1}\right)-\psi\left(d\left(x_{n-1}, x_{n+1}\right), 0\right) \\
& \leq \frac{1}{2} d\left(x_{n-1}, x_{n+1}\right) \\
& \leq \frac{1}{2}\left[d\left(x_{n-1}, x_{n}\right)+d\left(x_{n}, x_{n+1}\right)\right]
\end{aligned}
$$

and so $d_{n} \leq d_{n-1}$ for all $n \in \mathbb{N}$. Then there exist $d \geq 0$ such that $\lim _{n \rightarrow \infty} d_{n}=d$. Suppose, on the contrary, that $d>0$. Also, taking limit as $n \rightarrow \infty$ in (5), we deduce

$$
d \leq \frac{1}{2} \lim _{n \rightarrow \infty} d\left(x_{n-1}, x_{n+1}\right) \leq \frac{1}{2}(d+d)=d
$$

that is,

$$
\lim _{n \rightarrow \infty} d\left(x_{n-1}, x_{n+1}\right)=2 d
$$

Taking limit as $n \rightarrow \infty$ in (5) and using (6), we get

$$
d \leq \frac{1}{2}[2 d]-\psi(2 d, 0)
$$

Consequently, we have $\psi(2 d, 0)=0$, which yields $d=0$. Hence

$$
\lim _{n \rightarrow \infty} d\left(x_{n}, x_{n+1}\right)=0
$$

We shall show that $\left\{x_{n}\right\}$ is a Cauchy sequence. To reach this goal, first we prove the following claim:

(K) For every $\varepsilon>0$, there exists $n \in \mathbb{N}$ such that if $r, q \geq n$ with $r-q \equiv 1(m)$, then

$$
d\left(x_{r}, x_{q}\right)<\varepsilon \text {. }
$$


Suppose, to the contrary, that there exists $\varepsilon>0$ such that for any $n \in \mathbb{N}$, we can find $r_{n}>q_{n} \geq n$ with $r_{n}-q_{n} \equiv 1(m)$ satisfying

$$
d\left(x_{q_{n}}, x_{r_{n}}\right) \geq \varepsilon
$$

Now, we take $n>2 m$. Then, corresponding to $q_{n} \geq n$, one can choose $r_{n}$ in such a way that it is the smallest integer with $r_{n}>q_{n}$ satisfying $r_{n}-q_{n} \equiv 1(m)$ and $d\left(x_{q_{n}}, x_{r_{n}}\right) \geq \varepsilon$. Therefore, $d\left(x_{q_{n}}, x_{r_{n}-m}\right)<\varepsilon$. By using the triangular inequality,

$$
\varepsilon \leq d\left(x_{q_{n}}, x_{r_{n}}\right) \leq d\left(x_{q_{n}}, x_{r_{n}-m}\right)+\sum_{i=1}^{m} d\left(x_{r_{n}-i}, x_{r_{n}-i-1}\right)<\varepsilon+\sum_{i=1}^{m} d\left(x_{r_{n}-i}, x_{r_{n}-i-1}\right) .
$$

Letting $n \rightarrow \infty$ in the last inequality, keeping (7) in mind, we derive that

$$
\lim _{n \rightarrow \infty} d\left(x_{q_{n}}, x_{r_{n}}\right)=\varepsilon
$$

Again,

$$
\begin{aligned}
\varepsilon & \leq d\left(x_{q_{n}}, x_{r_{n}}\right) \\
& \leq d\left(x_{q_{n}}, x_{q_{n}+1}\right)+d\left(x_{q_{n}+1}, x_{r_{n}+1}\right)+d\left(x_{r_{n}+1}, x_{r_{n}}\right) \\
& \leq d\left(x_{q_{n}}, x_{q_{n}+1}\right)+d\left(x_{q_{n}+1}, x_{q_{n}}\right)+d\left(x_{q_{n}}, x_{r_{n}}\right)+d\left(x_{r_{n}}, x_{r_{n}+1}\right)+d\left(x_{r_{n}+1}, x_{r_{n}}\right) .
\end{aligned}
$$

Taking (7) and (9) into account, we get

$$
\lim _{n \rightarrow \infty} d\left(x_{q_{n}+1}, x_{r_{n}+1}\right)=\varepsilon
$$

as $n \rightarrow \infty$ in (9).

Also we have the following inequalities:

$$
d\left(x_{q_{n}}, x_{r_{n}+1}\right) \leq d\left(x_{q_{n}}, x_{r_{n}}\right)+d\left(x_{r_{n}}, x_{r_{n}+1}\right)
$$

and

$$
d\left(x_{q_{n}}, x_{r_{n}}\right) \leq d\left(x_{q_{n}}, x_{r_{n}+1}\right)+d\left(x_{r_{n}}, x_{r_{n}+1}\right) .
$$

Letting $n \rightarrow \infty$ in (11) and (12), we derive that

$$
\lim _{n \rightarrow \infty} d\left(x_{q_{n}}, x_{r_{n}+1}\right)=\varepsilon
$$

Again, we have

$$
d\left(x_{r_{n}}, x_{q_{n}+1}\right) \leq d\left(x_{r_{n}}, x_{r_{n}+1}\right)+d\left(x_{r_{n}+1}, x_{q_{n}+1}\right)
$$

and

$$
d\left(x_{r_{n}+1}, x_{q_{n}+1}\right) \leq d\left(x_{r_{n}+1}, x_{r_{n}}\right)+d\left(x_{r_{n}}, x_{q_{n}+1}\right) .
$$


Letting $n \rightarrow \infty$ in (14) and (15), we conclude that

$$
\lim _{n \rightarrow \infty} d\left(x_{r_{n}}, x_{q_{n}+1}\right)=\varepsilon
$$

Since $x_{q_{n}}$ and $x_{r_{n}}$ lie in different adjacently labeled sets $X_{i}$ and $X_{i+1}$ for certain $1 \leq i \leq m$, using the fact that $T$ is a cyclic weak $r-(\eta, \xi)$-C-contractive mapping of the first kind, we have

$$
\begin{aligned}
& r^{2} d\left(x_{r_{n}+1}, x_{q_{n}+1}\right) \\
& \quad=r^{2} d\left(T x_{r_{n}}, T x_{q_{n}}\right) \leq \eta\left(x_{r_{n}}\right) \eta\left(x_{q_{n}}\right) d\left(T x_{r_{n}}, T x_{q_{n}}\right) \\
& \quad \leq \xi\left(x_{r_{n}}\right) \xi\left(x_{q_{n}}\right)\left[\frac{1}{2}\left[d\left(x_{r_{n}}, T x_{q_{n}}\right)+d\left(x_{q_{n}}, T x_{r_{n}}\right)\right]-\psi\left(d\left(x_{r_{n}}, T x_{q_{n}}\right), d\left(x_{q_{n}}, T x_{r_{n}}\right)\right)\right] \\
& \quad \leq r^{2}\left[\frac{1}{2}\left[d\left(x_{r_{n}}, x_{q_{n}+1}\right)+d\left(x_{q_{n}}, x_{r_{n}+1}\right)\right]-\psi\left(d\left(x_{r_{n}}, x_{q_{n}+1}\right), d\left(x_{q_{n}}, x_{r_{n}+1}\right)\right)\right],
\end{aligned}
$$

which implies

$$
d\left(x_{r_{n}+1}, x_{q_{n}+1}\right) \leq \frac{1}{2}\left[d\left(x_{r_{n}}, x_{q_{n}+1}\right)+d\left(x_{q_{n}}, x_{r_{n}+1}\right)\right]-\psi\left(d\left(x_{r_{n}}, x_{q_{n}+1}\right), d\left(x_{q_{n}}, x_{r_{n}+1}\right)\right) .
$$

Letting $n \rightarrow \infty$ in the inequality above and keeping the expressions (7), (9), (10), (13), (16) in mind, we conclude that

$$
\varepsilon \leq \varepsilon-\psi(\varepsilon, \varepsilon)
$$

Thus, we have $\psi(\varepsilon, \varepsilon)=0$, which yields that $\varepsilon=0$. Hence, $(\mathrm{K})$ is satisfied.

We shall show that the sequence $\left\{x_{n}\right\}$ is Cauchy. Fix $\varepsilon>0$. By the claim, we find $n_{0} \in \mathbb{N}$ such that if $r, q \geq n_{0}$ with $r-q \equiv 1(m)$, then

$$
d\left(x_{r}, x_{q}\right) \leq \frac{\varepsilon}{2}
$$

Since $\lim _{n \rightarrow \infty} d\left(x_{n}, x_{n+1}\right)=0$, we also find $n_{1} \in \mathbb{N}$ such that

$$
d\left(x_{n}, x_{n+1}\right) \leq \frac{\varepsilon}{2 m}
$$

for any $n \geq n_{1}$. Suppose that $r, s \geq \max \left\{n_{0}, n_{1}\right\}$ and $s>r$. Then, there exists $k \in\{1,2, \ldots, m\}$ such that $s-r \equiv k(m)$. Therefore, $s-r+\varphi \equiv 1(m)$ for $\varphi=m-k+1$. So, we have, for $j \in\{1, \ldots, m\}, s+j-r \equiv 1(m)$

$$
d\left(x_{r}, x_{s}\right) \leq d\left(x_{r}, x_{s+j}\right)+d\left(x_{s+j}, x_{s+j-1}\right)+\cdots+d\left(x_{s+1}, x_{s}\right)
$$

By (18) and (19) and from the last inequality, we get

$$
d\left(x_{r}, x_{s}\right) \leq \frac{\varepsilon}{2}+j \times \frac{\varepsilon}{2 m} \leq \frac{\varepsilon}{2}+m \times \frac{\varepsilon}{2 m}=\varepsilon
$$

This proves that $\left\{x_{n}\right\}$ is a Cauchy sequence. Since $Y$ is closed in $(X, d)$, then $(Y, d)$ is also complete, there exists $x \in Y=\bigcup_{i=1}^{m} X_{i}$ such that $\lim _{n \rightarrow \infty} x_{n}=x$ in $(Y, d)$. In what follows, 
we prove that $x$ is a fixed point of $T$. In fact, since $\lim _{n \rightarrow \infty} x_{n}=x$ and, as $Y=\bigcup_{i=1}^{m} X_{i}$ is a cyclic representation of $Y$ with respect to $T$, the sequence $\left\{x_{n}\right\}$ has infinite terms in each $X_{i}$ for $i \in\{1,2, \ldots, m\}$. Suppose that $x \in X_{i}, T x \in X_{i+1}$ and we take a subsequence $x_{n_{k}}$ of $\left\{x_{n}\right\}$ with $x_{n_{k}} \in X_{i-1}$. Now from (iii) we have $\eta(x) \geq r$ and $\xi(x) \leq r$. By using the contractive condition, we can obtain

$$
\begin{aligned}
r^{2} d\left(T x, T x_{n_{k}}\right) & \leq \eta(x) \eta\left(x_{n_{k}}\right) d\left(T x, T x_{n_{k}}\right) \\
& \leq \xi(x) \xi\left(x_{n_{k}}\right)\left[\frac{1}{2}\left[d\left(x, T x_{n_{k}}\right)+d\left(x_{n_{k}}, T x\right)\right]-\psi\left(d\left(x, T x_{n_{k}}\right), d\left(x_{n_{k}}, T x\right)\right)\right] \\
& \leq r^{2}\left[\frac{1}{2}\left[d\left(x, T x_{n_{k}}\right)+d\left(x_{n_{k}}, T x\right)\right]-\psi\left(d\left(x, T x_{n_{k}}\right), d\left(x_{n_{k}}, T x\right)\right)\right],
\end{aligned}
$$

which implies

$$
d\left(T x, x_{n_{k}+1}\right) \leq \frac{1}{2}\left[d\left(x, x_{n_{k}+1}\right)+d\left(x_{n_{k}}, T x\right)\right]-\psi\left(d\left(x, x_{n_{k}+1}\right), d\left(x_{n_{k}}, T x\right)\right) .
$$

Passing to the limit as $k \rightarrow \infty$ in the last inequality, we get

$$
\begin{aligned}
d(x, T x) & \leq \frac{1}{2} d(x, T x)-\psi(0, d(x, T x)) \\
& \leq \frac{1}{2} d(x, T x)
\end{aligned}
$$

which implies $d(x, T x)=0$, i.e., $x=T x$. Finally, to prove the uniqueness of the fixed point, suppose that $x, y \in \operatorname{Fix}(T)_{Y}$ such that $\eta(x) \geq r, \eta(y) \geq r, \xi(x) \leq r, \xi(y) \leq r$, where $x \neq y$. The cyclic character of $T$ and the fact that $x, y \in X$ are fixed points of $T$ imply that $x, y \in \bigcap_{i=1}^{m} X_{i}$. Suppose that $x \neq y$. That is, $d(x, y)>0$. Using the contractive condition, we obtain

$$
\begin{aligned}
r^{2} d(T x, T y) & \leq \eta(x) \eta(y) d(T x, T y) \\
& \leq \xi(x) \xi(y)\left[\frac{1}{2}[d(x, T y)+d(y, T x)]-\psi(d(x, T y), d(y, T x))\right] \\
& \leq r^{2}\left[\frac{1}{2}[d(x, T y)+d(y, T x)]-\psi(d(x, T y), d(y, T x))\right],
\end{aligned}
$$

which implies

$$
d(x, y) \leq d(x, y)-\psi(d(x, y), d(x, y)) .
$$

Then $\psi(d(x, y), d(x, y))=0$ and so $d(x, y)=0$, i.e., $x=y$, which is a contradiction. This finishes the proof. 
Example 2.2 Let $X=\mathbb{R}$ with the metric $d(x, y)=|x-y|$ for all $x, y \in X$. Suppose $A_{1}=$ $(-\infty, 0]$ and $A_{2}=[0, \infty)$ and $Y=\bigcup_{i=1}^{2} A_{i}$. Define $T: Y \rightarrow Y$ and $\eta, \xi: Y \rightarrow[0, \infty)$ by

$$
\begin{aligned}
& T x= \begin{cases}\frac{x+9}{\sqrt{x^{8}+1}} & \text { if } x \in(-\infty,-10], \\
\frac{\sin ^{2} x}{x^{4}} & \text { if } x \in[-10,-5), \\
-3 x & \text { if } x \in[-5,-1), \\
0 & \text { if } x \in[-1,1], \\
-5 \ln x & \text { if } x \in(1,5), \\
\frac{4-x}{(3-x)(2-x)} & \text { if } x \in[5,10), \\
\sqrt[3]{9-x} & \text { if } x \in[10, \infty),\end{cases} \\
& \eta(x)= \begin{cases}4 & \text { if } x \in[-1,1], \\
0 & \text { otherwise }\end{cases} \\
& \xi(x)= \begin{cases}4 & \text { if } x \in[-1,1], \\
10 & \text { otherwise. }\end{cases}
\end{aligned}
$$

Also, define $\psi:[0, \infty)^{2} \rightarrow[0, \infty)$ by $\psi(t, s)=\frac{1}{4}(t+s)$. Clearly, $T A_{1} \subseteq A_{2}, T A_{2} \subseteq A_{1}$ and $\eta(0) \geq 4$ and $\xi(0) \leq 4$. Let $\eta(x) \geq 4$, then $x \in[-1,1]$. On the other hand, $T w \in[-1,1]$ for all $w \in[-1,1]$, i.e., $\eta(T x) \geq 1$. Similarly, $\xi(x) \leq 4$ implies $\xi(T x) \leq 4$. Therefore, $T$ is an $r-(\eta, \xi)$ admissible mapping. Let $\left\{x_{n}\right\}$ be a sequence in $X$ such that $\eta\left(x_{n}\right) \geq 1, \xi\left(x_{n}\right) \leq 1$ and $x_{n} \rightarrow x$ as $n \rightarrow \infty$. Then $x_{n} \in[-1,1]$. So, $x \in[-1,1]$, i.e., $\eta(x) \geq 1$ and $\xi(x) \leq 1$.

Let $x \in A_{1}$ and $y \in A_{2}$. Now, if $x \notin[-1,0]$ or $y \notin[0,1]$, then $\eta(x) \eta(y)=0$. Also, if $x \in[-1,0]$ and $y \in[0,1]$, then $d(T x, T y)=0$. That is, $\eta(x) \eta(y) d(T x, T y)=0$ for all $x \in A_{1}$ and all $y \in A_{2}$. Hence,

$$
\eta(x) \eta(y) d(T x, T y)=0 \leq \xi(x) \xi(y)\left[\frac{1}{2}[d(x, T y)+d(y, T x)]-\psi(d(x, T y), d(y, T x))\right]
$$

for all $x \in A_{1}$ and $y \in A_{2}$. Then $T$ is a cyclic weak $r-(\eta, \xi)$-C-contractive mapping of the first kind. Therefore all the conditions of Theorem 2.1 hold and $T$ has a fixed point in $A_{1} \cap A_{2}$. Here, $x=0$ is a fixed point of $T$.

Theorem 2.3 Let $(X, d)$ be a complete metric space, $m \in \mathbb{N}, X_{1}, X_{2}, \ldots, X_{m}$ be nonempty closed subsets of $(X, p)$ and $Y=\bigcup_{i=1}^{m} X_{i}$. Suppose that $T: Y \rightarrow Y$ is a cyclic weak $r-(\eta, \xi)$ $C$-contractive mapping of the second kind such that

(i) $T$ is $r-(\eta, \xi)$-admissible;

(ii) there exists $x_{0} \in Y$ such that $\eta\left(x_{0}\right) \geq r$ and $\xi\left(x_{0}\right) \leq r$;

(iii) if $\left\{x_{n}\right\}$ is a sequence in $Y$ such that $\eta\left(x_{n}\right) \geq r$ and $\xi\left(x_{n}\right) \leq r$ for all $n \in \mathbb{N}$ and $x_{n} \rightarrow x$ as $n \rightarrow \infty$, then $\eta(x) \geq r$ and $\xi(x) \leq r$.

Then $T$ has a fixed point $x \in \bigcap_{i=1}^{n} X_{i}$. Moreover, if $\eta(x) \geq r, \eta(y) \geq r, \xi(x) \leq r, \xi(y) \leq r$ for all $x, y \in F i x(T)_{Y}$, then $T$ has a unique fixed point.

Proof By a similar method as in the proof of Theorem 2.1, we have

$$
x_{n+1}=T x_{n}, \quad \eta\left(x_{n}\right) \geq r \quad \text { and } \quad \xi\left(x_{n}\right) \leq r \quad \text { for all } n \in \mathbb{N} \text {. }
$$


We shall show that the sequence $\left\{d_{n}:=d\left(x_{n}, x_{n+1}\right)\right\}$ is non-increasing. Due to (3) with $x=$ $x_{n-1}$ and $y=x_{n}$, we get

$$
\begin{aligned}
\left(r^{2}+r\right)^{d\left(x_{n}, x_{n+1}\right)} & =\left(r^{2}+r\right)^{d\left(T x_{n-1}, T x_{n}\right)} \leq\left(\eta\left(x_{n-1}\right) \eta\left(x_{n}\right)+r\right)^{d\left(T x_{n-1}, T x_{n}\right)} \\
& \leq\left(\xi\left(x_{n-1}\right) \xi\left(x_{n}\right)+r\right)^{\left[\frac{1}{2}\left[d\left(x_{n-1}, T x_{n}\right)+d\left(x_{n}, T x_{n-1}\right)\right]-\psi\left(d\left(x_{n-1}, T x_{n}\right), d\left(x_{n}, T x_{n-1}\right)\right)\right]} \\
& =\left(\xi\left(x_{n-1}\right) \xi\left(x_{n}\right)+r\right)^{\left[\frac{1}{2} d\left(x_{n-1}, x_{n+1}\right)-\psi\left(d\left(x_{n-1}, x_{n+1}\right), 0\right)\right]} \\
& \leq\left(r^{2}+r\right)^{\left[\frac{1}{2} d\left(x_{n-1}, x_{n+1}\right)-\psi\left(d\left(x_{n-1}, x_{n+1}\right), 0\right)\right]},
\end{aligned}
$$

which implies

$$
\begin{aligned}
d\left(x_{n}, x_{n+1}\right) & \leq \frac{1}{2} d\left(x_{n-1}, x_{n+1}\right)-\psi\left(d\left(x_{n-1}, x_{n+1}\right), 0\right) \\
& \leq \frac{1}{2} d\left(x_{n-1}, x_{n+1}\right) \\
& \leq \frac{1}{2}\left[d\left(x_{n-1}, x_{n}\right)+d\left(x_{n}, x_{n+1}\right)\right]
\end{aligned}
$$

and so $d_{n} \leq d_{n-1}$ for all $n \in \mathbb{N}$. Then there exists $d \geq 0$ such that $\lim _{n \rightarrow \infty} d_{n}=d$. We shall show that $d=0$ by the method of reductio ad absurdum. Suppose that $d>0$. By letting $n \rightarrow \infty$ in (22), we deduce

$$
d \leq \frac{1}{2} \lim _{n \rightarrow \infty} d\left(x_{n-1}, x_{n+1}\right) \leq \frac{1}{2}(d+d)=d,
$$

that is,

$$
\lim _{n \rightarrow \infty} d\left(x_{n-1}, x_{n+1}\right)=2 d
$$

Taking limit as $n \rightarrow \infty$ in (22) and using (23), we get

$$
d \leq \frac{1}{2}[2 d]-\psi(2 d, 0)
$$

Thus, we have $\psi(2 d, 0)=0$ and hence $d=0$, which is a contradiction. Consequently, we have

$$
\lim _{n \rightarrow \infty} d_{n}=\lim _{n \rightarrow \infty} d\left(x_{n}, x_{n+1}\right)=0 .
$$

We shall show that $\left\{x_{n}\right\}$ is a Cauchy sequence. To reach this goal, first we prove the following claim:

(K) For every $\varepsilon>0$, there exists $n \in \mathbb{N}$ such that if $r, q \geq n$ with $r-q \equiv 1(m)$, then

$$
d\left(x_{r}, x_{q}\right)<\varepsilon \text {. }
$$

Suppose, to the contrary, that there exists $\varepsilon>0$ such that for any $n \in \mathbb{N}$ we can find $r_{n}>q_{n} \geq n$ with $r_{n}-q_{n} \equiv 1(m)$ satisfying

$$
d\left(x_{q_{n}}, x_{r_{n}}\right) \geq \varepsilon .
$$


Following the related lines in Theorem 2.1, we deduce

$$
\begin{aligned}
& \lim _{n \rightarrow \infty} d\left(x_{q_{n}}, x_{r_{n}}\right)=\varepsilon, \\
& \lim _{n \rightarrow \infty} d\left(x_{q_{n}+1}, x_{r_{n}+1}\right)=\varepsilon, \\
& \lim _{n \rightarrow \infty} d\left(x_{q_{n}}, x_{r_{n}+1}\right)=\varepsilon
\end{aligned}
$$

and

$$
\lim _{n \rightarrow \infty} d\left(x_{r_{n}}, x_{q_{n}+1}\right)=\varepsilon
$$

Since $x_{q_{n}}$ and $x_{r_{n}}$ lie in different adjacently labeled sets $X_{i}$ and $X_{i+1}$ for certain $1 \leq i \leq m$, using the fact that a cyclic weak $r-(\eta, \xi)$-C-contractive mapping of the second kind, we have

$$
\begin{aligned}
\left(r^{2}+r\right)^{d\left(x_{r_{n}+1}, x_{q_{n}+1}\right)} & =\left(r^{2}+r\right)^{d\left(T x_{r_{n}}, T x_{q_{n}}\right)} \leq\left(\eta\left(x_{r_{n}}\right) \eta\left(x_{q_{n}}\right)+r\right)^{d\left(T x_{r_{n}}, T x_{q_{n}}\right)} \\
& \leq\left(\xi\left(x_{r_{n}}\right) \xi\left(x_{q_{n}}\right)+r\right)^{\left[\frac{1}{2}\left[d\left(x_{r_{n}}, T x_{q_{n}}\right)+d\left(x_{q_{n}}, T x_{r_{n}}\right)\right]-\psi\left(d\left(x_{r_{n}}, T x_{q_{n}}\right), d\left(x_{q_{n}}, T x_{r_{n}}\right)\right)\right]} \\
& \leq\left(r^{2}+r\right)^{\left[\frac{1}{2}\left[d\left(x_{r_{n}}, x_{q_{n}+1}\right)+d\left(x_{q_{n}}, x_{r_{n}+1}\right)\right]-\psi\left(d\left(x_{r_{n}}, x_{q_{n}+1}\right), d\left(x_{q_{n}}, T x_{r_{n}+1}\right)\right)\right],}
\end{aligned}
$$

which implies

$$
d\left(x_{r_{n}+1}, x_{q_{n}+1}\right) \leq \frac{1}{2}\left[d\left(x_{r_{n}}, x_{q_{n}+1}\right)+d\left(x_{q_{n}}, x_{r_{n}+1}\right)\right]-\psi\left(d\left(x_{r_{n}}, x_{q_{n}+1}\right), d\left(x_{q_{n}}, T x_{r_{n}+1}\right)\right) .
$$

Letting $n \rightarrow \infty$ in the inequality above and by applying (24) (26), (27), (28), (29), we deduce that

$$
\varepsilon \leq \varepsilon-\psi(\varepsilon, \varepsilon)
$$

Consequently, we have $\psi(\varepsilon, \varepsilon)=0$, and hence $\varepsilon=0$. As a result, we conclude that $(\mathrm{K})$ is satisfied. We assert that the sequence $\left\{x_{n}\right\}$ is Cauchy. Fix $\varepsilon>0$. By the claim, we find $n_{0} \in \mathbb{N}$ such that if $r, q \geq n_{0}$ with $r-q \equiv 1(m)$, then

$$
d\left(x_{r}, x_{q}\right) \leq \frac{\varepsilon}{2}
$$

Since $\lim _{n \rightarrow \infty} d\left(x_{n}, x_{n+1}\right)=0$, we also find $n_{1} \in \mathbb{N}$ such that

$$
d\left(x_{n}, x_{n+1}\right) \leq \frac{\varepsilon}{2 m}
$$

for any $n \geq n_{1}$. Suppose that $r, s \geq \max \left\{n_{0}, n_{1}\right\}$ and $s>r$. Then there exists $k \in\{1,2, \ldots, m\}$ such that $s-r \equiv k(m)$. Therefore, $s-r+\varphi \equiv 1(m)$ for $\varphi=m-k+1$. So, we have, for $j \in\{1, \ldots, m\}, s+j-r \equiv 1(m)$,

$$
d\left(x_{r}, x_{s}\right) \leq d\left(x_{r}, x_{s+j}\right)+d\left(x_{s+j}, x_{s+j-1}\right)+\cdots+d\left(x_{s+1}, x_{s}\right) .
$$


By (30) and (31) and from the last inequality, we get

$$
\begin{aligned}
d\left(x_{r}, x_{s}\right) & \leq \frac{\varepsilon}{2}+j \times \frac{\varepsilon}{2 m} \\
& \leq \frac{\varepsilon}{2}+m \times \frac{\varepsilon}{2 m}=\varepsilon .
\end{aligned}
$$

This proves that $\left\{x_{n}\right\}$ is a Cauchy sequence. Since $Y$ is closed in $(X, d)$, then $(Y, d)$ is also complete, there exists $x \in Y=\bigcup_{i=1}^{m} X_{i}$ such that $\lim _{n \rightarrow \infty} x_{n}=x$ in $(Y, d)$. In what follows, we prove that $x$ is a fixed point of $T$. In fact, since $\lim _{n \rightarrow \infty} x_{n}=x$ and, as $Y=\bigcup_{i=1}^{m} X_{i}$ is a cyclic representation of $Y$ with respect to $T$, the sequence $\left\{x_{n}\right\}$ has infinite terms in each $X_{i}$ for $i \in\{1,2, \ldots, m\}$. Suppose that $x \in X_{i}, T x \in X_{i+1}$ and we take a subsequence $x_{n_{k}}$ of $\left\{x_{n}\right\}$ with $x_{n_{k}} \in X_{i-1}$. Now from (iii) we have $\eta(x) \geq r$ and $\xi(x) \leq r$. By using the contractive condition, we can obtain

$$
\begin{aligned}
\left(r^{2}+r\right)^{d\left(T x, T x_{n_{k}}\right)} & \leq\left(\eta(x) \eta\left(x_{n_{k}}\right)+r\right)^{d\left(T x, T x_{n_{k}}\right)} \\
& \leq\left(\xi(x) \xi\left(x_{n_{k}}\right)+r\right)^{\left[\frac{1}{2}\left[d\left(x, T x_{n_{k}}\right)+d\left(x_{n_{k}}, T x\right)\right]-\psi\left(d\left(x, T x_{n_{k}}\right), d\left(x_{n_{k}}, T x\right)\right)\right]} \\
& \leq\left(r^{2}+r\right)^{\left[\frac{1}{2}\left[d\left(x, T x_{n_{k}}\right)+d\left(x_{n_{k}}, T x\right)\right]-\psi\left(d\left(x, T x_{n_{k}}\right), d\left(x_{n_{k}}, T x\right)\right)\right]},
\end{aligned}
$$

which implies

$$
d\left(T x, x_{n_{k}+1}\right) \leq \frac{1}{2}\left[d\left(x, x_{n_{k}+1}\right)+d\left(x_{n_{k}}, T x\right)\right]-\psi\left(d\left(x, x_{n_{k}+1}\right), d\left(x_{n_{k}}, T x\right)\right) .
$$

Passing to the limit as $k \rightarrow \infty$ in the last inequality, we get

$$
d(x, T x) \leq \frac{1}{2} d(x, T x)-\psi(0, d(x, T x)) \leq \frac{1}{2} d(x, T x)
$$

which implies $d(x, T x)=0$, i.e., $x=T x$. Finally, to prove the uniqueness of the fixed point, suppose that $x, y \in \operatorname{Fix}(T)_{Y}$ such that $\eta(x) \geq r, \eta(y) \geq r, \xi(x) \leq r, \xi(y) \leq r$, where $x \neq y$. The cyclic character of $T$ and the fact that $x, y \in X$ are fixed points of $T$ imply that $x, y \in \bigcap_{i=1}^{m} X_{i}$. Suppose that $x \neq y$. That is, $d(x, y)>0$. Using the contractive condition, we obtain

$$
\begin{aligned}
\left(r^{2}+r\right)^{d(T x, T y)} & \leq(\eta(x) \eta(y)+r)^{d(T x, T y)} \\
& \leq(\xi(x) \xi(y)+r)^{\left[\frac{1}{2}[d(x, T y)+d(y, T x)]-\psi(d(x, T y), d(y, T x))\right]} \\
& \leq\left(r^{2}+r\right)^{\left[\frac{1}{2}[d(x, T y)+d(y, T x)]-\psi(d(x, T y), d(y, T x))\right]},
\end{aligned}
$$

which implies

$$
d(x, y) \leq d(x, y)-\psi(d(x, y), d(x, y)) .
$$

Hence, we obtain $\psi(d(x, y), d(x, y))=0$, which implies $d(x, y)=0$, that is, $x=y$ a contradiction. 


\section{Existence of solutions of an integral equation}

For $T>0$, we denote by $X=C([0, T])$ the set of real continuous functions on $[0, T]$. We endow $X$ with the metric

$$
d_{\infty}(u, v)=\|u-v\|_{\infty} \quad \text { for all } u, v \in X .
$$

It is evident that $\left(X, d_{\infty}\right)$ is a complete metric space.

Consider the integral equation

$$
u(t)=\int_{0}^{T} G(t, s) f(s, u(s)) d s \quad \text { for all } t \in[0, T]
$$

(1) $f:[0, T] \times \mathbb{R} \rightarrow \mathbb{R}$ and $G:[0, T] \times[0, T] \rightarrow[0, \infty)$ are continuous functions.

(2) Let $(\alpha, \beta) \in X^{2},\left(\alpha_{0}, \beta_{0}\right) \in \mathbb{R}^{2}$ such that

$$
\alpha_{0} \leq \alpha(t) \leq \beta(t) \leq \beta_{0} \quad \text { for all } t \in[0, T]
$$

Assume that for all $t \in[0, T]$, we have

$$
\alpha(t) \leq \int_{0}^{T} G(t, s) f(s, \beta(s)) d s
$$

and

$$
\beta(t) \geq \int_{0}^{T} G(t, s) f(s, \alpha(s)) d s .
$$

Let for all $s \in[0, T], f(s, \cdot)$ be a decreasing function, that is,

$$
x, y \in \mathbb{R}, \quad x \geq y \quad \Longrightarrow \quad f(s, x) \leq f(s, y) .
$$

Let $Z:=\{u \in X: u \leq \beta\} \cup\{u \in X: u \geq \alpha\}$. There exist $0 \leq r<1$ and $\theta, \pi: Z \rightarrow \mathbb{R}$ such that if $\theta(x) \geq 0$ and $\theta(y) \geq 0$ with $\left(x \leq \beta_{0}\right.$ and $\left.y \geq \alpha_{0}\right)$ or $\left(x \geq \alpha_{0}\right.$ and $\left.y \leq \beta_{0}\right)$, then for every $s \in[0, T]$, we have

$$
|f(s, x(s))-f(s, y(s))| \leq \frac{r|\pi(y)|}{2}(|x(s)-T y(s)|+|y(s)-T x(s)|) .
$$

(3) Assume that

$$
\left\|\int_{0}^{T}|\pi(y)| G(t, s) d s\right\|_{\infty} \leq 1
$$

for all $x \in Z$, where $\theta(x) \geq 0$. Suppose that

$$
\theta(x) \geq 0 \quad \Longrightarrow \quad \theta(T x) \geq 0 \quad \text { for } x \in\{u \in X: u \leq \beta\} \cup\{u \in X: u \geq \alpha\}
$$

(4) If $\left\{x_{n}\right\}$ is a sequence in $\{u \in X: u \leq \beta\} \cup\{u \in X: u \geq \alpha\}$ such that $\theta\left(x_{n}\right) \geq 0$ for all $n \in \mathbb{N}$ and $x_{n} \rightarrow x$ as $n \rightarrow \infty$, then $\theta(x) \geq 0$.

(5) There exists $x_{0} \in\{u \in X: u \leq \beta\} \cup\{u \in X: u \geq \alpha\}$ such that $\theta\left(x_{0}\right) \geq 0$. 
Theorem 3.1 Under assumptions (1)-(5), integral equation (33) has a solution in $\{u \in$ $C([0, T]): \alpha(t) \leq u(t) \leq \beta(t)$ for all $t \in[0, T]\}$.

Proof Define the closed subsets of $X, A_{1}$ and $A_{2}$ by

$$
A_{1}=\{u \in X: u \leq \beta\}
$$

and

$$
A_{2}=\{u \in X: u \geq \alpha\} .
$$

Also define the mapping $T: X \rightarrow X$ by

$$
T u(t)=\int_{0}^{T} G(t, s) f(s, u(s)) d s \quad \text { for all } t \in[0, T] .
$$

Let us prove that

$$
T\left(A_{1}\right) \subseteq A_{2} \quad \text { and } \quad T\left(A_{2}\right) \subseteq A_{1} .
$$

Suppose $u \in A_{1}$, that is,

$$
u(s) \leq \beta(s) \text { for all } s \in[0, T] .
$$

Applying condition (37), since $G(t, s) \geq 0$ for all $t, s \in[0, T]$, we obtain that

$$
G(t, s) f(s, u(s)) \geq G(t, s) f(s, \beta(s)) \quad \text { for all } t, s \in[0, T] .
$$

The above inequality with condition (35) imply that

$$
\int_{0}^{T} G(t, s) f(s, u(s)) d s \geq \int_{0}^{T} G(t, s) f(s, \beta(s)) d s \geq \alpha(t)
$$

for all $t \in[0, T]$. Then we have $T u \in A_{2}$.

Similarly, let $u \in A_{2}$, that is,

$$
u(s) \geq \alpha(s) \quad \text { for all } s \in[0, T] .
$$

Using condition (37), since $G(t, s) \geq 0$ for all $t, s \in[0, T]$, we obtain that

$$
G(t, s) f(s, u(s)) \leq G(t, s) f(s, \alpha(s)) \quad \text { for all } t, s \in[0, T] .
$$

The above inequality with condition (36) imply that

$$
\int_{0}^{T} G(t, s) f(s, u(s)) d s \leq \int_{0}^{T} G(t, s) f(s, \alpha(s)) d s \leq \beta(t)
$$

for all $t \in[0, T]$. Then we have $T u \in A_{1}$. Also, we deduce that (41) holds. 
Now, let $(u, v) \in A_{1} \times A_{2}$, that is, for all $t \in[0, T]$,

$$
u(t) \leq \beta(t), \quad v(t) \geq \alpha(t)
$$

This implies from condition (34) that for all $t \in[0, T]$,

$$
u(t) \leq \beta_{0}, \quad v(t) \geq \alpha_{0} .
$$

Now, by conditions (39) and (38), we have, for all $s \in[0, T]$,

$$
\begin{aligned}
|T u(t)-T v(t)| & =\left|\int_{0}^{T} G(t, s)[f(s, u(s))-f(s, v(s))] d s\right| \\
& \leq \int_{0}^{T} G(t, s)|f(s, u(s))-f(s, v(s))| d s \\
& \leq \int_{0}^{T} G(t, s) \frac{r|\pi(y)|}{2}(|u(s)-T v(s)|+|v(s)-T u(s)|) d s \\
& \leq \frac{r}{2}\left(\|u-T v\|_{\infty}+\|v-T u\|_{\infty}\right)\left\|\int_{0}^{T}|\pi(v)| G(t, s) d s\right\|_{\infty} \\
& \leq \frac{r}{2}\left(\|u-T v\|_{\infty}+\|v-T u\|_{\infty}\right),
\end{aligned}
$$

which implies

$$
\|T u-T v\|_{\infty} \leq \frac{r}{2}\left(\|u-T v\|_{\infty}+\|v-T u\|_{\infty}\right) .
$$

Define $\eta, \xi: Z \rightarrow[0, \infty)$ by $\eta(u)=\left\{\begin{array}{ll}1, & \theta(u) \geq 0, \\ 0, & \text { otherwise }\end{array}\right.$ and $\xi(u)=1$. Further, $\psi\left(t_{1}, t_{2}\right)=\frac{(1-r)}{2}\left(t_{1}+t_{2}\right)$. Hence,

$$
\eta(u) \eta(v) d_{\infty}(T u, T v) \leq \frac{r}{2}\left(d_{\infty}(u, T v)+d_{\infty}(v, T u)\right)
$$

for all $(u, v) \in A_{1} \times A_{2}$. By a similar method, we can show that the above inequality holds if $(u, v) \in A_{2} \times A_{1}$. Now, all the conditions of Theorem 2.1 hold and $T$ has a fixed point $z^{*}$ in

$$
A_{1} \cap A_{2}=\{u \in C([0, T]): \alpha \leq u(t) \leq \beta \text { for all } t \in[0, T]\} .
$$

That is, $z^{*} \in A_{1} \cap A_{2}$ is the solution to (33).

Example 3.2 In this example, we denote by $X=C([0,1])$ the set of real continuous functions on $[0,1]$. We endow $X$ with the metric

$$
d_{\infty}(u, v)=\|u-v\|_{\infty} \quad \text { for all } u, v \in X .
$$


Consider the following continuous functions:

$$
f(t, x)=\left\{\begin{array}{ll}
x^{3} & \text { if } x \in(-\infty, 0), \\
0 & \text { if } x \in[0,1] \\
x^{2}-1 & \text { if } x \in(1,4), \\
15 & \text { if } x \in\left[4, \sqrt{e^{16}-1}\right], \\
x^{2}+16-e^{16} & \text { if } x \in\left(\sqrt{e^{16}-1}, \infty\right)
\end{array} \quad \text { for all } t \in[0,1]\right.
$$

and

$$
G(t, s)=\frac{t}{1+t} e^{s} \quad \text { for all } s, t \in[0,1] \times[0,1]
$$

Let $\alpha(t)=0$ and $\beta(t)=1$. Then, for $\left(\alpha_{0}, \beta_{0}\right)=(0,1) \in \mathbb{R}^{2}$, we have

$$
\begin{aligned}
& \alpha_{0} \leq \alpha(t) \leq \beta(t) \leq \beta_{0} ; \\
& \alpha(t)=0 \leq \int_{0}^{1} G(t, s) f(s, \beta(s)) d s=0
\end{aligned}
$$

and

$$
\beta(t)=1 \geq \int_{0}^{1} G(t, s) f(s, \alpha(s)) d s=0 .
$$

Also, $Z:=\{u \in X: u \leq \beta\} \cup\{u \in X: u \geq \alpha\}=X$. Define $\theta, \pi: Z \rightarrow \mathbb{R}$ by

$$
\theta(x(t))=\left\{\begin{array}{ll}
0 & \text { if } 0 \leq x(t) \leq 1 \text { for all } t \in[0,1] \\
-1, & \text { otherwise }
\end{array} \text { and } \quad \pi(x)=\frac{1}{e-1} .\right.
$$

Clearly, $\theta(0) \geq 0$. Also, if $\theta(x(t)) \geq 0$, then $0 \leq x(t) \leq 1$. On the other hand,

$$
T u(t)=\int_{0}^{1} G(t, s) f(s, u(s)) d s=0
$$

for all $0 \leq u(t) \leq 1$. That is, $\theta(T x(t)) \geq 0$. Hence, $\theta(x) \geq 0$ implies $\theta(T x) \geq 0$.

Assume $\theta(x(s)) \geq 0$ and $\theta(y(s)) \geq 0$ with $\left(x \leq \beta_{0}\right.$ and $\left.y \geq \alpha_{0}\right)$ or $\left(x \geq \alpha_{0}\right.$ and $\left.y \leq \beta_{0}\right)$.

Thus, $0 \leq x(s) \leq 1$ and $0 \leq y(s) \leq 1$, which implies $f(s, x(s))=f(s, y(s))=0$. That is,

$$
|f(s, x(s))-f(s, y(s))|=0 \leq \frac{r|\pi(y)|}{2}(|x(s)-T y(s)|+|y(s)-T x(s)|)
$$

for all $s \in[0,1]$, where $0 \leq r<1$. Further,

$$
\int_{0}^{1}|\pi(y)| G(t, s) d s=\int_{0}^{1} \frac{1}{e-1} \frac{t}{1+t} e^{s} d s=\frac{t}{1+t} \leq 1,
$$

and so

$$
\left\|\int_{0}^{T}|\pi(y)| G(t, s) d s\right\|_{\infty} \leq 1
$$


Assume that $\left\{x_{n}\right\}$ is a sequence in $X$ such that $\theta\left(x_{n}\right) \geq 0$ for all $n \in \mathbb{N}$ and $x_{n} \rightarrow x$ as $n \rightarrow \infty$.

Then $0 \leq x_{n} \leq 1$. So, $0 \leq x \leq 1$. That is, $\theta(x) \geq 0$.

Therefore, all of the conditions of Theorem 3.1 are satisfied. Then the integral equation

$$
u(t)=\frac{t}{1+t} \int_{0}^{1} e^{s} f(s, u(s)) d s
$$

has a solution in $\{u \in C([0,1]): 0 \leq u(t) \leq 1$ for all $t \in[0,1]\}$. Here, $u(t)=0$ is a solution.

But if we chose $x_{0}(t)=0$ and $y_{0}(t)=\sqrt{e^{16}-1}$, then $f\left(s, x_{0}(s)\right)=0$ and $f\left(s, y_{0}(s)\right)=15$. That

is,

$$
\left|f\left(s, x_{0}(s)\right)-f\left(s, y_{0}(s)\right)\right|=15 \text {. }
$$

Also,

$$
\sqrt{\ln \left(\left|x_{0}(s)-y_{0}(s)\right|^{2}+1\right)}=\sqrt{\ln \left(\left|0-\sqrt{e^{16}-1}\right|^{2}+1\right)}=\sqrt{\ln e^{16}}=4,
$$

and so

$$
\left|f\left(s, x_{0}(s)\right)-f\left(s, y_{0}(s)\right)\right|=15>4=\sqrt{\ln \left(\left|x_{0}(s)-y_{0}(s)\right|^{2}+1\right)} .
$$

That is, Theorem 3.1 of [6] cannot be applied to this example.

\section{Competing interests}

The authors declare that they have no competing interests.

Authors' contributions

All authors contributed equally and significantly in writing this article. All authors read and approved the final manuscript.

\section{Author details}

${ }^{1}$ Department of Mathematics, Cumhuriyet University, Sivas, Turkey. ${ }^{2}$ Department of Mathematics, Atilim University, Incek, Ankara 06836, Turkey. ${ }^{3}$ Faculty of Sciences and Mathematics, University of Nis, Visegradska 33, Nis, 18000, Serbia. ${ }^{4}$ Young Researchers and Elite Club, Rasht Branch, Islamic Azad University, Rasht, Iran.

\section{Acknowledgements}

The authors thank the anonymous referees for their remarkable comments, suggestions and ideas that helped to improve this paper. The third author (V Rakocević) is supported by Grant No. 174025 of the Ministry of Science,

Technology and Development, Republic of Serbia

Received: 14 May 2013 Accepted: 18 October 2013 Published: 11 Nov 2013

\section{References}

1. Alghamdi, MA, Hussain, N, Salimi, P: Fixed point and coupled fixed point theorems on b-metric-like spaces. J. Inequal. Appl. 2013, 402 (2013)

2. Harandi, AA, Emami, H: A fixed point theorem for contraction type maps in partially ordered metric spaces and application to ordinary differential equations. Nonlinear Anal. 72, 2238-2242 (2010)

3. Nieto, JJ, Lopez, RR: Contractive mapping theorems in partially ordered sets and applications to ordinary differential equations. Order 22, 223-239 (2005)

4. Harjani, J, Sadarangani, K: Fixed point theorems for weakly contractive mappings in partially ordered sets. Nonlinear Anal. 71, 3403-3410 (2009)

5. Moradi, S, Karapinar, E, Aydi, H: Existence of solutions for a periodic boundary value problem via generalized weakly contractions. Abstr. Appl. Anal. 2013, Article ID 704160 (2013)

6. Nahsine, HK: Cyclic generalized $\psi$-weakly contractive mappings and fixed point results with applications to integral equations. Nonlinear Anal. 75, 6160-6169 (2012)

7. Karapinar, E, Shatanawi, W: On weakly $(C, \psi, \phi)$-contractive mappings in partially ordered metric spaces. Abstr. Appl. Anal. 2012, Article ID 495892 (2012)

8. Karapinar, E, Yuce, IS: Fixed point theory for cyclic generalized weak $\phi$-contraction on partial metric spaces. Abstr Appl. Anal. 2012, Article ID 491542 (2012) 
9. Karapinar, E: Best proximity points of Kannan type cyclic weak $\phi$-contractions in ordered metric spaces. An. Univ. "Ovidius" Constanţa, Ser. Mat. 20(3), 51-64 (2012)

10. Karapinar, E: Best proximity points of cyclic mappings. Appl. Math. Lett. 25(11), 1761-1766 (2012)

11. Karapinar, E, Erhan, ÝM: Cyclic contractions and fixed point theorems. Filomat 26(4), 777-782 (2012)

12. Karapýnar, E: Fixed point theory for cyclic weak $\phi$-contraction. Appl. Math. Lett. 24(6), 822-825 (2011)

13. Karapinar, E, Rakocevic, V: On cyclic generalized weakly C-contractions on partial metric spaces. Abstr. Appl. Anal. 2013, Article ID 831491 (2013)

14. Salimi, P, Latif, A, Hussain, N: Modified $\alpha-\psi$-contractive mappings with applications. Fixed Point Theory Appl. 2013 $151(2013)$

15. Karapinar, E, Nashine, HK: Fixed point theorem for cyclic weakly Chatterjea type contractions. J. Appl. Math. 2012, Article ID 165698 (2012)

16. Karapinar, E, Salimi, P: Dislocated metric space to metric spaces with some fixed point theorems. Fixed Point Theory Appl. 2013, 222 (2013)

17. Moradlou, F, Salimi, P, Vetro, P: Fixed point results for $r$ - $(\boldsymbol{\eta}, \boldsymbol{\xi}, \psi)$-contractive mappings of type (I), (II) and (III). Filomat 27, 403-410 (2013)

18. Kirk, WA, Srinavasan, PS, Veeramani, P: Fixed points for mapping satisfying cyclical contractive conditions. Fixed Point Theory 4, 79-89 (2003)

10.1186/1029-242X-2013-529

Cite this article as: Gülyaz et al.: Existence of a solution of integral equations via fixed point theorem. Journal of Inequalities and Applications 2013, 2013:529

\section{Submit your manuscript to a SpringerOpen ${ }^{\circ}$ journal and benefit from:}

- Convenient online submission

- Rigorous peer review

- Immediate publication on acceptance

- Open access: articles freely available online

- High visibility within the field

- Retaining the copyright to your article 